\title{
A first approach to the distortion analysis of nonlinear analog circuits utilizing $\mathrm{X}$-parameters
}

\author{
H. Weber, C. Widemann, and W. Mathis \\ Institute four Theoretical Electrical Engineering, Leibniz Universität Hannover, Hanover, Germany \\ Correspondence to: C. Widemann (widemann@tet.uni-hannover.de)
}

\begin{abstract}
In this contribution a first approach to the distortion analysis of nonlinear 2-port-networks with $\mathrm{X}$ parameters ${ }^{1}$ is presented. The X-parameters introduced by Verspecht and Root (2006) offer the possibility to describe nonlinear microwave 2-port-networks under large signal conditions. On the basis of X-parameter measurements with a nonlinear network analyzer (NVNA) behavioral models can be extracted for the networks. These models can be used to consider the nonlinear behavior during the design process of microwave circuits. The idea of the present work is to extract the behavioral models in order to describe the influence of interfering signals on the output behavior of the nonlinear circuits. Hereby, a simulator is used instead of a NVNA to extract the X-parameters. Assuming that the interfering signals are relatively small compared to the nominal input signal, the output signal can be described as a superposition of the effects of each input signal. In order to determine the functional correlation between the scattering variables, a polynomial dependency is assumed. The required datasets for the approximation of the describing functions are simulated by a directional coupler model in Cadence Design Framework. The polynomial coefficients are obtained by a least-square method. The resulting describing functions can be used to predict the system's behavior under certain conditions as well as the effects of the interfering signal on the output signal.
\end{abstract}

\section{Introduction}

A high-frequency circuit can be designed by describing the $\mathrm{N}$-port using scattering parameters (S-parameters) that allow to describe the N-port without current and voltage. Instead alternative variables are used. These variables are called the scattering variables and they are linear combination of the

\footnotetext{
${ }^{1} \mathrm{X}$-parameter is a registered trademark of Agilent Technologies,
}

voltage and current of the analyzed $\mathrm{N}$-port. The scattering variables are proportional to the electrical power and are measurable for high-frequencies. However, the S-parameters are restricted to linear and time-invariant N-ports. Thus, they cannot describe nonlinear circuits under large signal excitation. In the last two decades, the description with Sparameters was extended by Verspecht (1995). The linear S-parameters are advanced to the so called X-parameters introduced by Agilent Technologies (Verspecht and Root, 2006). The basic principle is called the polyharmonic distortion (PHD) which allows to describe a nonlinear circuit under large signal excitation. Moreover, it is possible to extract the X-parameters using a nonlinear vector analyzer (NVNA) (Roblin, 2011). By this means, a behavioral model for the device under test (DUT) can be developed. This model can be used for the design (Peláez Pérez, 2012) or can be added as additional information in the data sheet of an IC producer (Horn et al., 2008). The PHD assumes that only one large signal is applied to the $\mathrm{N}$-port which defines the large signal operating point (LSOP). Other signals are superimposed at an integer multiple of the large signal frequency and are assumed as small signals. In this paper, a method is presented for the extraction of X-parameters using a simulator like Cadence. Therefore, the extension of scattering variables for nonlinear circuits is reviewed at first.

\section{Scattering variables}

Before describing a nonlinear N-port with X-parameters, the scattering variables are introduced. Therefore, the behavior of scattering variables for a nonlinear circuit have to be examined. For this purpose, the 1-port in Fig. 1 is given.

The nonlinear impedance in the 1-port is described by

$i=\alpha_{1} u+\varepsilon \alpha_{3} u^{3}$. 


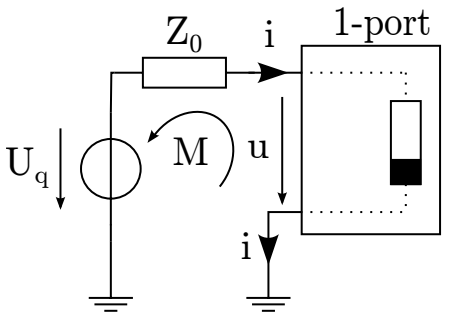

Fig. 1. Nonlinear resistor regarded as 1-port.

Table 1. Labeling of the scattering variables for a nonlinear 1-port.

\begin{tabular}{ll}
\hline Scattering Variable & \\
\hline$b_{11}$ & $A \sin (\omega t)\left(\frac{1-\alpha_{1} Z_{0}-\frac{3}{4} A^{2} \varepsilon \alpha_{3}}{2 \sqrt{Z_{0}}}\right)$ \\
$b_{13}$ & $A^{3} \sin (3 \omega t) \frac{Z_{0} \varepsilon \alpha_{3}}{4 \cdot 2 \sqrt{Z_{0}}}$ \\
$a_{11}$ & $\frac{U_{q}}{2 \sqrt{Z_{0}}}$ \\
\hline
\end{tabular}

Furthermore the mesh

$u=U_{q}-Z_{0} \cdot i$

can be set up. Assuming the port voltage to be

$u=A \sin (\omega t)$

one can insert these equations into the definitions of the scattering variables

$a:=\frac{u+Z_{0} \cdot i}{2 \sqrt{\left(Z_{0}\right)}}=\frac{U_{q}}{2 \sqrt{Z_{0}}}$

and with Eq. (1)

$$
\begin{aligned}
b: & =\frac{u-Z_{0} \cdot i}{2 \sqrt{\left(Z_{0}\right)}}=\frac{\left(1-Z_{0} \alpha_{1}\right) u-Z_{0} \varepsilon \alpha_{3} u^{3}}{2 \sqrt{Z_{0}}} \\
& =A \sin (\omega t)\left(\frac{1-\alpha_{1} Z_{0}-\frac{3}{4} A^{2} \varepsilon \alpha_{3}}{2 \sqrt{Z_{0}}}\right) \\
& +A^{3} \sin (3 \omega t) \frac{Z_{0} \varepsilon \alpha_{3}}{4 \cdot 2 \sqrt{Z_{0}}} .
\end{aligned}
$$

In Eq. (4), one can see that the scattering variable $a$ is independent from the connected nonlinear 1-port. In contrast, the scattering variable $b$ depends on the connected nonlinear 1-port. Due to the nonlinearity, harmonics can be recognized in Eq. (5). In order to describe this nonlinear effect with the scattering variables, an extended notation is introduced in Table 1 . In addition to the first index, a second one is established to describe the generated harmonics separately according to the fundamental.

In Fig. 2, the scattering variables are represented in the frequency domain in order to illustrate the harmonic frequencies.

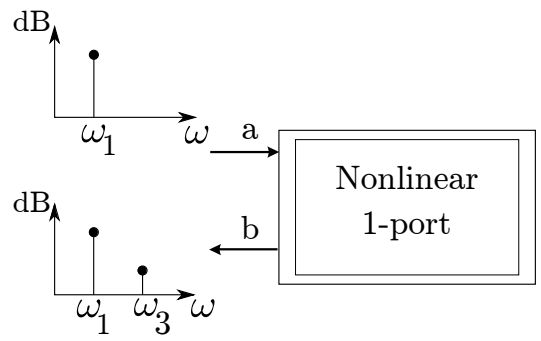

Fig. 2. Scattering variables represented in the frequency domain for a nonlinear 1-port.

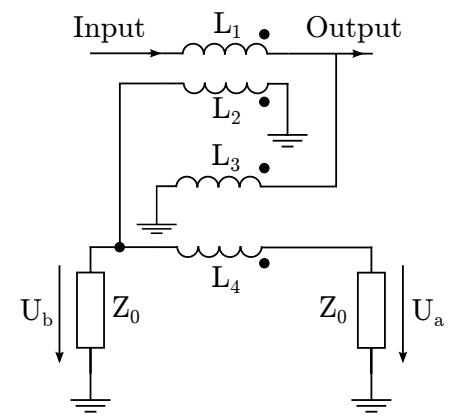

Fig. 3. Directional coupler model to obtain the scattering variables $a$ and $b$.

Using the established notation for the scattering variables, it is now possible to describe all frequencies. However, it is still complicated to simulate these scattering variables because they are a linear combination of voltage and current and cannot be measured or simulated in the circuit directly. Therefore, a directional coupler is used in a real environment to determine all scattering variables. In the next section a directional coupler model is illustrated to provide all advantages of a real directional coupler in a simulator.

\subsection{Directional coupler model}

In the measurement technology, a directional coupler is used to obtain the scattering variables $a$ and $b$. For simulation purpose, there are model approaches to the directional coupler behavior. One possibility of such a model is presented in Fig. 3 (Sierra, 2010).

This model consists of two coupled inductor pairs to transfer the current and the voltage which are used to determine the scattering variables $a$ and $b$. The dimension of the inductor pairs can be determined by the coupling factor

$C=20 \log _{10}\left(\frac{N_{2}}{N_{1}}\right)$.

$N_{2}$ and $N_{1}$ represent the number of windings of the coupled inductors $L_{1}$ and $L_{2}$, respectively. By the relation

$\frac{N_{2}}{N_{1}}=\sqrt{\frac{L_{2}}{L_{1}}}$ 
the inductors $L_{1}$ and $L_{2}$ can be dimensioned. Furthermore, $L_{3}=L_{2}$ and $L_{4}=L_{1}$ are assumed. The scattering variables are represented in this model by the voltages $U_{a}$ and $U_{b}$. In order to determine the scattering variables, the voltages have to be multiplied with $\frac{1}{\sqrt{Z_{0}}}$. In the following, this model will be used to extract the scattering variables in order to describe a nonlinear N-port with X-parameters.

\section{Extension to X-parameter}

Certain properties of a linear N-port can be characterized by the S-parameters. Considering the scattering variables the Sparameters describe the connection between these by

$$
\left(\begin{array}{c}
b_{1} \\
b_{2} \\
\vdots \\
b_{N}
\end{array}\right)=\left(\begin{array}{cccc}
S_{11} & S_{12} & \cdots & S_{1 j} \\
S_{21} & S_{22} & \cdots & S_{2 j} \\
\vdots & \vdots & \ddots & \vdots \\
S_{N 1} & S_{N 2} & \cdots & S_{N N}
\end{array}\right) \cdot\left(\begin{array}{c}
a_{1} \\
a_{2} \\
\vdots \\
a_{N}
\end{array}\right)
$$

Furthermore, the behavior of the nonlinear system depends on the excitation. Considering only one generated frequency, the relation between the scattering variable $a_{i j}$ and $b_{n m}$ can be described by

$b_{n m}=F_{n m}\left(a_{11}, a_{12}, \ldots a_{21}, a_{22}, \ldots\right)$.

The nonlinear scattering function $F_{n m}(\cdot)$ is hard to identify (Sun et al., 2010). However, under certain circumstances, simplifications can be applied. One assumption is that only one of the input signals represented by the scattering variables $a_{j k}$ is a large signal which is typically defined as $a_{11}$. The rest of the signals occur at integer multiples of the large signal frequency with a small amplitude. Considering these assumptions, the system is linearized (Verspecht et al., 2005). Therefore, the superposition principle is valid for the small signals which are superimposed to the LSOP. The principle of the linearization is illustrated in Fig. 4. This allows to reduce the Eq. (9) to

$$
\begin{aligned}
b_{n m}=\sum_{j k} & S_{n j, m k}\left(\left|a_{11}\right|\right) P^{+m-k} a_{j k} \\
& +\sum_{j k} T_{n j, m k}\left(\left|a_{11}\right|\right) P^{+m+k} a_{j k}^{*}
\end{aligned}
$$

with $P=e^{j \varphi_{a_{11}}}$ for the phase normalization (Verspecht and Root, 2006). The functions $S_{n j, m k}\left(\left|a_{11}\right|\right)$ and $T_{n j, m k}\left(\left|a_{11}\right|\right)$ are called X-parameters.

For linear systems, the parameters $S_{n j, m k}\left(\left|a_{11}\right|\right)$ are reduced to the linear scattering parameters in Eq. (8). Due to the two tone excitation, intermodulation occurs in nonlinear systems. This behavior is represented by the parameters $T_{n j, m k}\left(\left|a_{11}\right|\right)$ that is hence only necessary for nonlinear systems. Exploiting Eq. (10) and considering the restriction for $a_{j k} \neq a_{11}$ to be small signals, it is possible to calculate the behavior of the $\mathrm{N}$-port for multiple input signals.

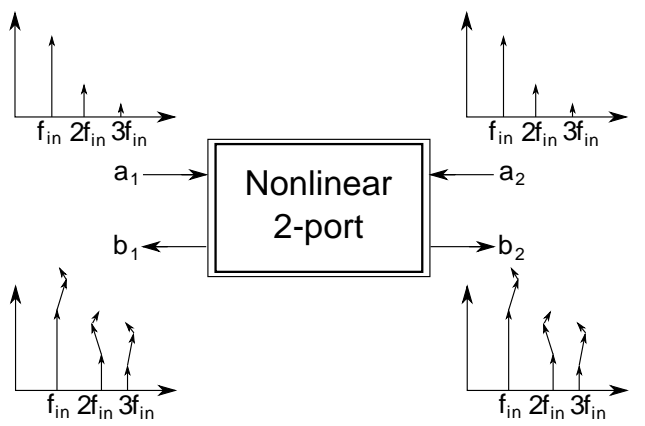

Fig. 4. Superposition for a nonlinear system (Verspecht and Root, 2006).

\subsection{Determine X-parameters with a simulator}

The X-parameters are extracted in the electrical measurement for a nonlinear N-port using a NVNA. In this section, a method to extract the $\mathrm{X}$-parameters by using a simulator like Cadence Spectre is going to be presented. For this purpose, the network is realized in the simulator. The required scattering variables are extracted by the directional coupler model introduced in Sect. 2.1. The structure for the extraction of Xparameters is exemplarily illustrated for a 2-port network in Fig. 5.

At first, the $\mathrm{N}$-port is excited only by the large signal $a_{11}$. Due to the phase normalization, the X-parameters $T_{n 1, m 1}\left(\left|a_{11}\right|\right)$ and $S_{n 1, m 1}\left(\left|a_{11}\right|\right)$ in Eq. (10) can be summarized to $S_{n 1, m 1}\left(\left|a_{11}\right|\right)$. Therefore, Eq. (10) is reduced to

$b_{n m}=S_{n 1, m 1}\left(\left|a_{11}\right|\right) P^{m}\left|a_{11}\right|$.

The X-parameters $S_{n 1, m 1}\left(\left|a_{11}\right|\right)$ can be determined by using the scattering variables $b_{n m}$ and $a_{11}$. Hence, pairs of values of the scattering variable $b_{n m}$ depending on $a_{11}$ are necessary. For a certain range of $\left|a_{11}\right|$, the amplitude and phase of the scattering variable $b_{n m}$ are determined. The pairs of values are interpolated by the least-square-fit (Herrmann, 2007).

After determining the X-parameters of the large signal, the $\mathrm{X}$-parameters for multiply inputs $\left(S_{n j, m k}\left(\left|a_{11}\right|\right), k>1\right)$ are determined. Therefore, further signals are used in addition to the large signal. A harmonic balance simulation allows to separate the intermodulation products which are represented by the X-parameters of the small signal. Exemplarily, one can assume that the excitation occurs at the frequencies $f_{a_{11}}=$ $200 \mathrm{MHz}$ and $f_{a_{12}}=400 \mathrm{MHz}$. If all harmonics of the small signal at the frequency $400 \mathrm{MHz}$ are neglected it results in three intermodulation products

$$
\begin{aligned}
1 \cdot f_{a_{11}}+0 \cdot f_{a_{12}} & =200 \mathrm{MHz}, \\
(-1) \cdot f_{a_{11}}+1 \cdot f_{a_{12}} & =200 \mathrm{MHz}, \\
3 \cdot f_{a_{11}}-1 \cdot f_{a_{12}} & =200 \mathrm{MHz}
\end{aligned}
$$

for the scattering variable $b_{n 1}$ at the frequency $200 \mathrm{MHz}$. Each of these intermodulation products is represented by a 


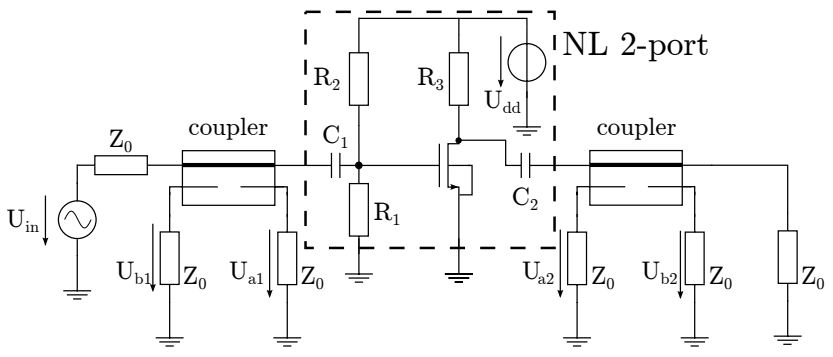

Fig. 5. Structure for the extraction of X-parameters for a 2-port.

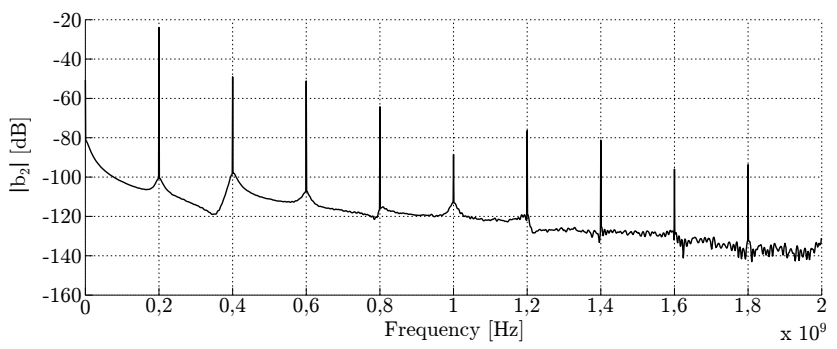

Fig. 6. Frequency domain of the scattering variable $b_{2}$.

$\mathrm{X}$-parameter. Thus, it is possible to determine each of them separately. The intermodulation product in Eq. (13) is represented by $S_{n 1,12}\left(\left|a_{11}\right|\right)$. In Eq. (14), one can see that the frequency of the small signal frequency of $f_{a_{12}}$ is multiplied by $(-1)$. Since this factor implies the complex conjugate signal $a_{12}^{*}$, the intermodulation product Eq. (14) is represented by $T_{n 1,12}\left(\left|a_{11}\right|\right)$. As functional approach for the X-parameters a polynomial approach is used for the interpolation.

\subsection{Description of nonlinear 2-port with X-parameters}

In the following, the nonlinear 2-port in Fig. 5 is described by using X-parameters. This example is limited to the description of the scattering variable $b_{21}$ in order to reduce the effort.

At first, the nonlinear 2-port is excited by $a_{11}$ at the frequency $f_{a_{11}}=200 \mathrm{MHz}$. Two directional couplers are used to extract all scattered variables. Exemplarily, the spectrum of scattering variables $\left|a_{1}\right|$ and $\left|b_{2}\right|$ are illustrated in Figs. 6 and 7. In the latter, one can see that also harmonics occur in addition to the excitation at $200 \mathrm{MHz}$. However, as in Eq. (4) calculated, the scattering variable $a_{1}$ depends only on the excitation. Nevertheless, harmonics are still generated due to the imperfection of the directional coupler. In the following, these harmonics are neglected because the amplitudes are very small. In Fig. 6, harmonic frequencies can be observed in the spectrum of the scattering variable $b_{2}$ which depends on the nonlinear 2-port as stated in Sect. 2.

In the following, the extraction of the X-parameters of the scattering variable $b_{21}$ will be executed. Therefore, the nonlinear 2-port is only excited by $a_{11}$ at first. Pairs of values of

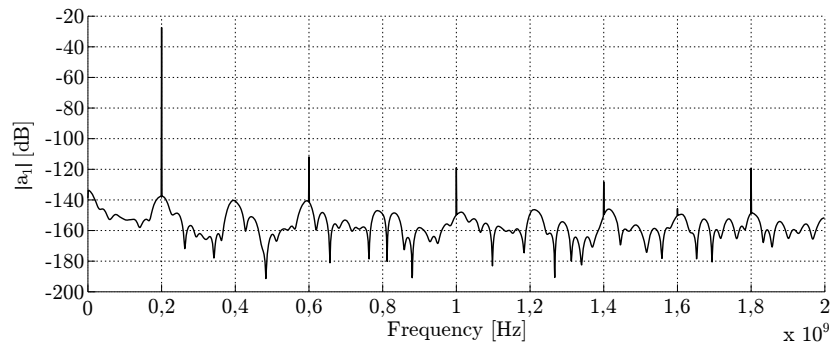

Fig. 7. Frequency domain of the scattering variable $a_{1}$.

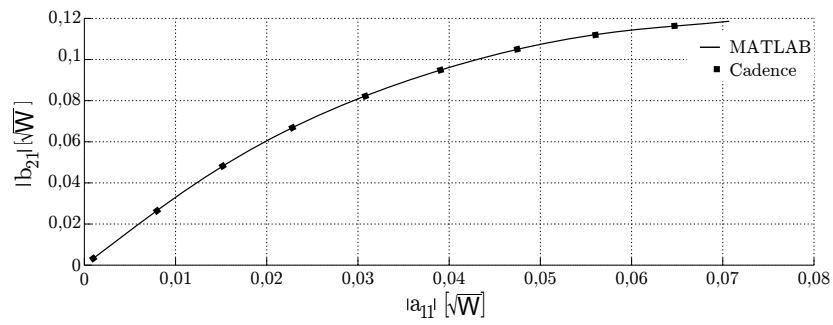

Fig. 8. Scattering variable $b_{21}$ simulated and calculated.

$a_{11}$ and $b_{21}$ are simulated and discrete values of the connecting X-parameter is calculated by

$S_{21,11}\left(\left|a_{11}\right|\right)=\frac{b_{21}}{a_{11}}$

These values are fitted by a polynomial approach which leads to an approximation of the X-parameter $S_{21,11}\left(\left|a_{11}\right|\right)$. The exemplary result is shown in Fig. 8 with the discrete simulation values. After determining the X-parameter $S_{21,11}\left(\left|a_{11}\right|\right)$, the small signal $a_{12}$ is superimposed at the frequency of 400 MHz. In this case, for the scattering variable $b_{21}$ one can say

$$
\begin{gathered}
b_{21}=S_{21,11}\left(\left|a_{11}\right|\right) a_{11}+S_{21,12}\left(\left|a_{11}\right|\right) P^{-1} a_{12} \\
+T_{21,12}\left(\left|a_{11}\right|\right) P^{3} a_{12}^{*} .
\end{gathered}
$$

The X-parameters $S_{21,12}\left(\left|a_{11}\right|\right)$ and $T_{21,12}\left(\left|a_{11}\right|\right)$ are determined by a harmonic balance simulation. At first, the contribution of the intermodulation product in Eq. (13) to the scattering variable $b_{21}$ is calculated. As for the X-parameter $S_{21,11}\left(\left|a_{11}\right|\right)$ the pairs of values are fitted by a polynomial approach which represents the X-parameter $S_{21,12}\left(\left|a_{11}\right|\right)$ as well as $S_{21,11}\left(\left|a_{11}\right|\right)$. The third intermodulation product in Eq. (14) is represented by $T_{21,12}\left(\left|a_{11}\right|\right)$. As $S_{21,12}\left(\left|a_{11}\right|\right)$ the $\mathrm{X}$-parameter $T_{21,12}\left(\left|a_{11}\right|\right)$ is determined by a harmonic balance and polynomial approach.

In Fig. 9, the simulated (dots) and calculated (plane) scattering variable $b_{21}$ is illustrated depending on $a_{11}$ and $a_{12}$. For small amplitudes of $a_{12}$ the calculated approximation coincides with the simulation whereas for higher amplitudes of $a_{12}$ one can see a deviation. This behavior is more obvious in the two dimensional plot in Fig. 10. Therefore, the 


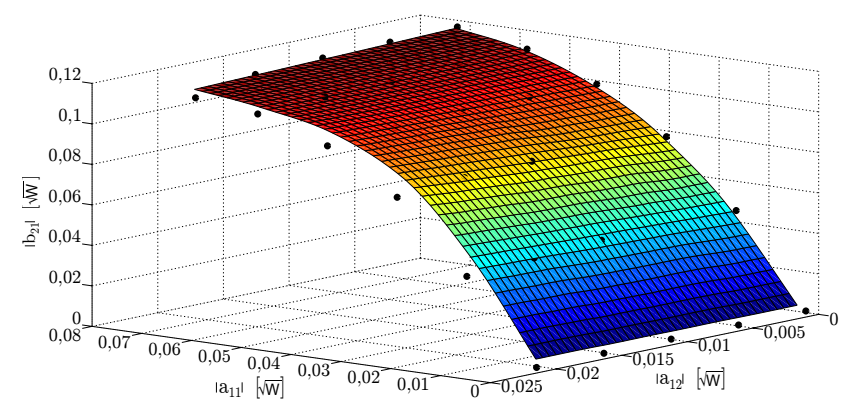

Fig. 9. Scattering variable $b_{21}$ depending on $a_{11}$ and $a_{12}$.

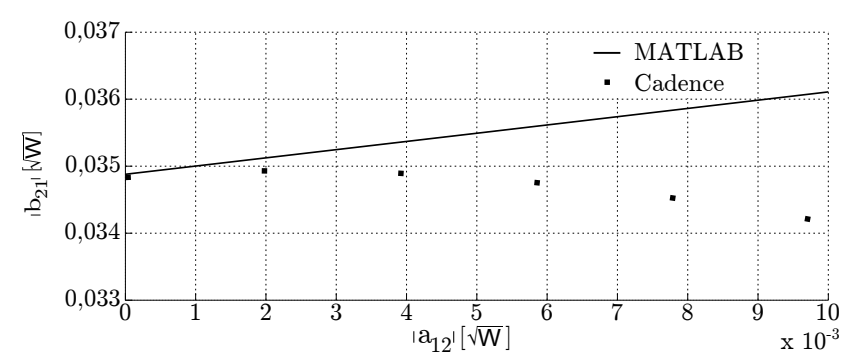

Fig. 10. Scattering variable $b_{21}$ depending on $a_{12}$ for fix $\left|a_{11}\right|=$ $0.011 \sqrt{W}$.

approximation is only valid for small amplitudes of the superimposed signals as assumed for the linearization of Eq. (9). In order to demonstrate the difference between simulation and approximation the relative error is calculated by

rel. error $=\frac{\left|b_{\mathrm{sim}}-b_{\mathrm{cal}}\right|}{\left|b_{\mathrm{sim}}\right|} \cdot 100[\%]$.

In Fig. 11, the relative error at discrete simulation points is illustrated for the results shown in Fig. 10. A regression line is added to illustrate the tendency. In Fig. 11, it can be seen that the smaller the amplitude of $a_{12}$, the better the approximation. However, the relative error raises drastically with increasing amplitude of $a_{12}$ due to the breach of the assumption of small $a_{j k} \neq a_{11}$.

\section{Conclusion and outlook}

In this paper, the description of nonlinear systems with $\mathrm{X}$ parameters using a circuit simulator like CADENCE Design Framework is presented. Therefore, a short introduction of the application of scattering variables on a nonlinear system was given. For the extraction of the scattering variables by a simulator a directional coupler model was used. After introducing a signal description of the nonlinear N-port with scattering variables, the $\mathrm{X}$-parameters were determined. The basic idea of X-parameters allows to linearize a nonlinear system under a large signal excitation. Furthermore, a method to extract the X-parameters by a simulator was demonstrated

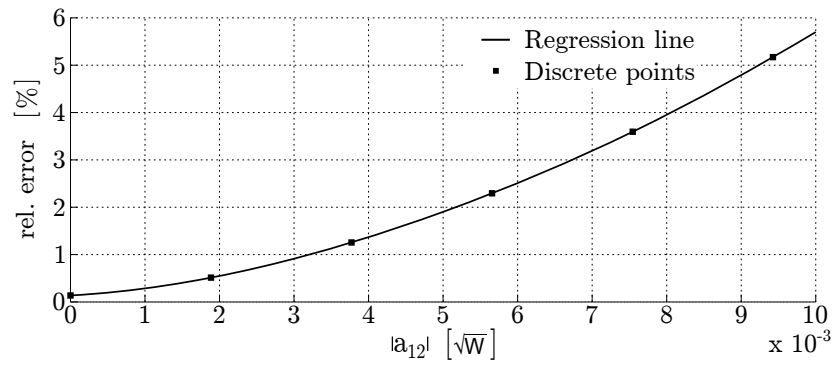

Fig. 11. Relative error of the approximation of $b_{21}$.

and the procedure was illustrated by an example. Future work will deal with an extension of Eq. (9) to

$$
\begin{aligned}
b_{n m}= & \sum_{j k} S_{n j, m k}\left(\left|a_{11}\right|\right) P^{+m-k} a_{j k}^{\mu} \\
& +\sum_{j k} T_{n j, m k}\left(\left|a_{11}\right|\right) P^{+m+k} a_{j k}^{* \mu} .
\end{aligned}
$$

It will be examined if the parameter $\mu$ can be determined by additional simulations. Considering this new parameter, the approximation probably covers a larger value range of $a_{j k}$.

\section{References}

Herrmann, N.: Höhere Mathematik, Oldenbourg Wissensch. Vlg., 2007.

Horn, J., Verspecht, J., Gunyan, D., Betts, L., Root, D., and Eriksson, J.: X-Parameter Measurement and Simulation of a GSM Handset Amplifier, in: Microwave Integrated Circuit Conference, 2008, EuMIC 2008. European, 135-138, 2008.

Peláez Pérez, A. M.: X-Parameters Based Analytical Design of Non-Linear Microwave Circuits: Application to Oscillator Design, Ph.D. thesis, Universidad Politécnica De Madrid, 2012.

Roblin, P.: Nonlinear RF Circuits and Nonlinear Vector Network Analyzers: Interactive Measurement and Design Techniques, Cambridge University Press, 2011.

Sierra, C. S.: Microwave directional couplers, 2010.

Sun, G., Xu, Y., and Liang, A.: The study of nonlinear scattering functions and X-parameters, in: Microwave and Millimeter Wave Technology (ICMMT), 2010 International Conference on, 10861089, 2010.

Verspecht, J.: Calibration of a Measurement System for High Frequency Nonlinear Devices, Ph.D. thesis, VRIJE UNIVERSITEIT BRUSSEL, 1995.

Verspecht, J. and Root, D. E.: Polyharmonic distortion modeling, Microwave Magazine IEEE, 2006.

Verspecht, J., Williams, D. F., Schreurs, D., Remley, K., and McKinley, M. D.: Linearization of large-signal scattering functions, Microwave Theory and Techniques, IEEE Transactions, 2005. 